\title{
Respuestas \\ Año 16 \\ Efecto de las calizas agregadas a las pastas empleadas en la fabricación de productos cerámicos de construcción del área metropolitana de Cúcuta
}

\author{
John Freddy Gelves Diaz ${ }^{1}$ | Jorge Sánchez Molina ${ }^{1}$ | Juan Isidro Díaz García
}

Recibido:

Enero 15 de 2011

Aceptado: Mayo 21 de 2011

${ }^{1}$ Centro de Investigación de Materiales Cerámicos, Grupo de Investigación en Tecnología Cerámica GITEC, Universidad Francisco de Paula Santander, Cúcuta-Colombia. E-Mail: johngelves@yahoo.es; j_sanchezmolina@yahoo.es;

${ }^{2}$ Universidad Nacional Experimental del Táchira, Dpto. Ing. Mecánica, San Cristóbal, Venezuela.

\section{Resumen}

El presente trabajo fue desarrollado para determinar los efectos generados por las calizas en las principales variables de control del proceso de producción de artículos cerámicos de construcción no esmaltados que se fabrican en la zona metropolitana de San José de Cúcuta, haciendo uso de la atmósfera oxidante en el proceso de cocción y la técnica de extrusión para su conformado. La investigación se llevó a cabo utilizando una muestra de arcilla y una muestra con alto contenido de sílice provenientes del municipio de Los Patios, Norte de Santander y rocas calizas provenientes del corregimiento de La don Juana jurisdicción del municipio de Bochalema. Se elaboraron diferentes mezclas aumentando el contenido de calizas a las cuales se les sometió al proceso de moldeo utilizando una extrusora de laboratorio manual, las probetas obtenidas fueron sometidas posteriormente a un proceso de secado en estufa de laboratorio hasta una temperatura de $100^{\circ} \mathrm{C}$, la cocción se llevó a cabo por un periodo comprendido entre 6 y 8 horas en un horno eléctrico en un rango de temperatura comprendido entre $980^{\circ} \mathrm{C}$ y $1100^{\circ} \mathrm{C}$, aumentando en gradientes de temperatura de 20 grados centígrados. Al final del proceso se realizaron pruebas para determinar la absorción de agua de las probetas cocidas. La experimentación realizada permitió establecer que las piedras calizas empleadas y adicionadas a las arcillas de la región utilizando la técnica de extrusión como método de conformado contribuyen a una mayor fusión del material respecto a las mezclas tradicionales en adiciones en peso inferiores al 5\%, siendo esto evidente a temperaturas superiores a los $1020^{\circ} \mathrm{C}$, como resultado de la mayor fusión bajo las condiciones establecidas, la adición de piedras calizas no mejora el proceso de control dimensional de los artículos cerámicos sometidos a cocción.

Palabrasclave: Absorción de agua, calizas, cerámica, contracción, eflorescencias, pastas. 


\section{Abstract}

The present work was developed to determine the effects generated by limestones in the principal variables of control of the process of production of ceramic articles of construction not enameled that are made in the Zona Metropolitana de Cúcuta, using the atmosphere oxidizer in the process of firing and the technology of extrusion for his shaped one. The investigation was carried out using a sample of clay and a sample with high place contained of silica from the municipality of Los Patios, Norte de Santander and limy rocks from the locality of La don Juana jurisdiction of Bochalema municipality. Different mixtures were elaborated increasing the content of limestones to which one submitted them to the process of shaped using an extruder of manual laboratory, the samples obtained were submitted later to a process of dried in laborator stove up to a temperature of $100^{\circ} \mathrm{C}$, the firing was carried out by a period understood between 6 and 8 hours in an electrical oven in a range of temperature understood between $980^{\circ} \mathrm{C}$ and $1100^{\circ} \mathrm{C}$, increasing in gradients of temperature of 20 centigrade degrees. At the end of the process tests were realized to determine the water absorption of the sintered samples. The realized experimentation allowed to establish that the limy stones used and added to the clays of the zone using the technology of extrusion as method of shaped, they contribute to a major merger of the material with regard to the traditional mixtures in additions in weight lower than $5 \%$ being evident this to temperatures superior to $1020^{\circ} \mathrm{C}$, as result of the major merger under the established conditions, the addition of limy stones they do not facilitate the process of dimensional control of the ceramic articles submitted to firing.

Keywords: Absorption of water, limestones, ceramics, contraction, efflorescences, pastes.

\section{Introducción}

L as calizas son rocas sedimentarías compuestas principalmente de carbonato de calcio y en menor grado de carbonato de magnesio, su principal uso es en la industria cementera, pero también tiene gran aplicabilidad en otros sectores entre estos las industria cerámica [1].

Las calizas agregadas a las pastas cerámicas se descomponen entre los $600^{\circ} \mathrm{C}$ y $900^{\circ} \mathrm{C}$ de acuerdo a la concentración de cada uno de sus componentes, el resultado de esta reacción es la formación de dióxido de carbono y óxidos de calcio y magnesio. El $\mathrm{MgO}$ y $\mathrm{CaO}$ así formados reaccionan con la sílice presente en la arcilla, dando $\mathrm{MgSiO}_{3}$ (silicato de magnesio) y $\mathrm{CaSiO}_{3}$ (silicato de calcio o pseudowollastonita). "Los cristales de silicato de calcio o magnesio constituyen una especie de esqueleto o estructura cristalina interna que aumenta la resistencia a la deformación a la pieza cocida, así como su resistencia mecánica en frío" [2].

Los materiales arcillosos ubicados en la zona metropolitana de Cúcuta se encuentran dentro del grupo de yacimientos de tipo sedimentario, las principales formaciones geológicas de donde se extrae los materiales 
para la fabricación de piezas cerámicas son el grupo Guayabo y la formación León, estas materias primas desde el punto de vista químico poseen un bajo contenido de carbonatos y un contenido de óxidos alcalinos que contribuyen a una mas rápida vitrificación del material cargado en el horno. Gran parte de la producción de piezas cerámicas de construcción no barnizadas ni esmaltadas elaboradas en la región son pisos y revestimientos [3-4], los cuales debido al modelo productivo empleado (Moldeo por extrusión y cocción en hornos de llama invertida) presentan un nivel de tolerancia dimensional muy elevado [5-6].

Aunque en Cúcuta y su área metropolitana se ha trabajado de manera empírica en el tema de la tolerancia dimensional de las piezas fabricadas, no existen referentes bibliográficos, que demuestren el efecto de las rocas calizas en el control dimensional de las piezas y en otras variables de control como la absorción de agua, el peso y la temperatura optima de cocción. La presente investigación se ha realizado para conocer mas a fondo el comportamiento que tienen las calizas al ser adicionadas a las pastas cerámicas, con miras de poder potencializar este recurso abundante en la región y contribuir a dar solución a los problemas de tolerancia dimensional de las piezas cerámicas elaboradas.

\section{Metodología}

La investigación fue desarrollada en la zona metropolitana de San José de Cúcuta, Norte de Santander. El muestreo utilizado fue de tipo intencionado, Las muestras de arcilla y de alto contenido de silice se obtuvo de las reservas de producción de una empresa ubicada en la vereda Llanitos parte alta, jurisdicción del municipio de Los Patios, en la periferia del casco urbano, material proveniente de las minas Tapias y Sigma respectivamente, la muestra de caliza se obtuvo del corregimiento de La don Juana jurisdicción del municipio de Bochalema. La investigación realizada es de tipo exploratoria y experimental la cual se llevó a cabo en las instalaciones del Centro de Investigación de Materiales Cerámicos CIMAC. Las principales características químicas y mineralógicas de la arcilla empleada son las que se muestran en el cuadro 1, las cuales fueron realizadas por Ferro Corporation utilizando la técnica de fluorescencia y difracción de rayos X [7], de forma similar en el cuadro 2, se puede apreciar la clasificación del tamaño de partícula de este material así como del compuesto con alto contenido de silice, estos datos fueron obtenidos empleando la técnica del hidrómetro según lo reportado por el autor [8]. Estos aspectos son básicos para el establecimiento de la aptitud cerámica de las materias primas.

Cuadro 1. Análisis químico y mineralógico de la arcilla empleada

\begin{tabular}{|c|c|c|c|}
\hline \multicolumn{2}{|c|}{ Análisis Químico } & \multicolumn{2}{|c|}{ Análisis Mineralógico } \\
\hline Compuesto & Promedio (\% peso) & Compuesto & Promedio (\% peso) \\
\hline $\mathrm{SiO}_{2}$ & 66.8 & \multirow{2}{*}{ Cuarzo } & \multirow{2}{*}{29.9} \\
\hline $\mathrm{Al}_{2} \mathrm{O}_{3}$ & 18.3 & & \\
\hline $\mathrm{Fe}_{2} \mathrm{O}_{3}$ & 4.5 & \multirow{2}{*}{ Caolinita } & \multirow{2}{*}{0.6} \\
\hline $\mathrm{TiO}_{2}$ & 0.5 & & \\
\hline $\mathrm{CaO}$ & 0.5 & \multirow{2}{*}{ Gismonidita } & \multirow{2}{*}{44.0} \\
\hline $\mathrm{MgO}$ & 0.6 & & \\
\hline $\mathrm{Na}_{2} \mathrm{O}$ & 0.3 & \multirow{3}{*}{ Illita } & \multirow{3}{*}{27.4} \\
\hline $\mathrm{K}_{2} \mathrm{O}$ & 1.5 & & \\
\hline $\mathrm{Ppc}$ & 7.0 & & \\
\hline
\end{tabular}

Cuadro 2. Tamaño de partícula de las muestras de arcilla y sílice (Aptitud cerámica)

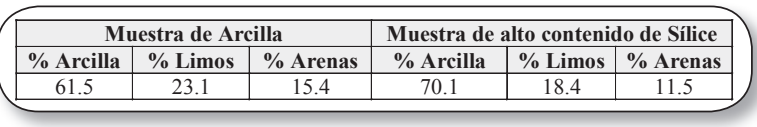

Respecto a la caliza empleada el cuadro 3 muestra el análisis químico de este material el cual fue desarrollado por la técnica de fluorescencia de rayos $\mathrm{X}$ de acuerdo a lo reportado por los autores [9].

Cuadro 3. Análisis químico de la caliza de La Don Juana

\begin{tabular}{|l|c|}
\hline \multicolumn{2}{|c|}{ Muestra Bochalema (\%) } \\
\hline $\mathrm{SiO}_{2}$ & 9.88 \\
\hline $\mathrm{Al}_{2} \mathrm{O}_{3}$ & 0.66 \\
\hline $\mathrm{Fe}_{2} \mathrm{O}_{3}$ & 2.24 \\
\hline $\mathrm{CaO}$ & 48.60 \\
\hline $\mathrm{MgO}$ & 0.30 \\
\hline $\mathrm{SO}_{3}$ & 0.04 \\
\hline Perdidas por calcinación & 38.28 \\
\hline
\end{tabular}


Efecto de las calizas agregadas a las pastas empleadas en la fabricación de productos cerámicos de construcción del área metropolitana de Cúcuta.

Para la realización de la investigación se contó con una extrusora de laboratorio manual (ver figura 1), con una boquilla de forma cuadrada, un molino de martillos de laboratorio marca Servitech, molinera rápida de laboratorio marca Gabbrielli, modelo CT058, estufa de secado marca "Dies" cuyo calentamiento se hace con resistencia eléctrica, tamiz malla ASTM 12, Horno mufla modelo 10/12 marca Gabbrielli, Balanzas digitales y un calibrador digital marca Mitutoyo.

Figura 1. Registro fotográfico de la extrusora empleada

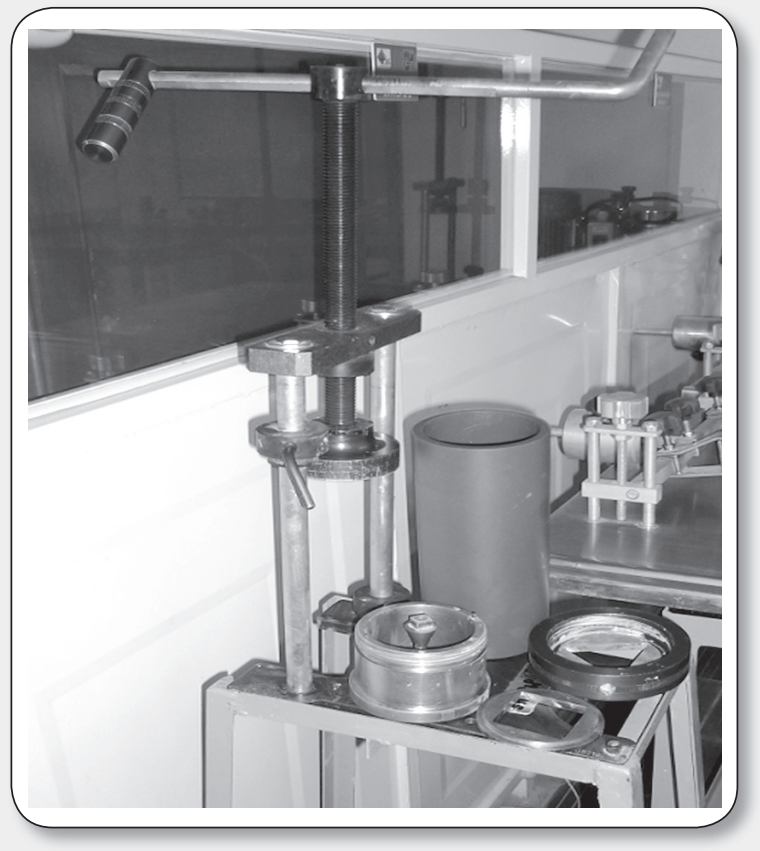

Los materiales recolectados fueron sometidos al proceso de reducción de tamaño de partícula, la muestra de arcilla y sílice se molturaron por vía seca en el molino de martillos y en el caso de la caliza por vía húmeda en la molinera rápida de laboratorio durante un tiempo de 20 minutos.

El material de arcilla y de alto contenido en Sílice fue sometido al proceso de tamizado, utilizando para ello la malla ASTM 12, medida típica en la industria cerámica regional. La Caliza se tamizo en una malla ASTM 100. Posterior a esto se prepararon varias mezclas o pastas homogenizadas de manera manual y en proporciones definidas de acuerdo a la información mostrada en el cuadro 4.
Cuadro 4. Mezclas realizadas en la investigación

ISSN 0122-820X (En peso)

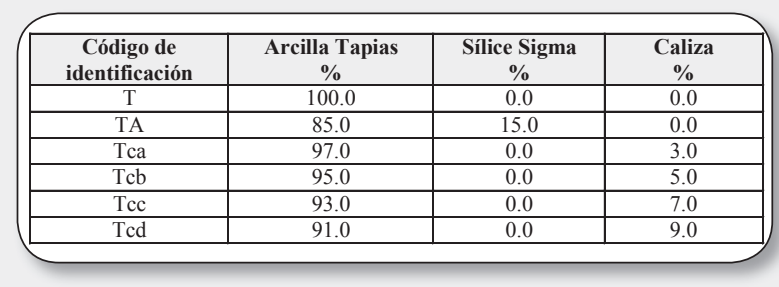

Una vez homogenizadas las mezclas se procedió a la humectación de las mismas con un $20 \%$ de agua en peso. A cada mezcla humectada se le realizó el proceso de conformado por extrusión, el corte se realizó de manera manual generándose probetas en forma de baldosas a las cuales en su superficie se les realizó una marca de 3,0 cm de largo para de determinar las contracciones de secado y cocción.

Las probetas conformadas se llevaron a una estufa de secado de resistencia eléctrica a temperatura ambiente $\left(30^{\circ} \mathrm{C}\right)$, durante las primeras cinco horas del proceso se realizó incrementos de temperatura de $10^{\circ} \mathrm{C} /$ hora, finalmente se llevo a la temperatura de $100^{\circ} \mathrm{C}$ hasta completar 24 horas de secado, se tomaron lecturas de peso y longitud de la marca de $3,0 \mathrm{~cm}$ realizada antes de entrar al secado, posteriormente las probetas fueron llevadas al horno mufla con calentamiento a través de resistencia eléctrica sometiendo las probetas a las temperaturas de cocción de $980^{\circ} \mathrm{C}, 1000^{\circ} \mathrm{C}, 1020^{\circ} \mathrm{C}, 1040^{\circ} \mathrm{C}, 1060^{\circ} \mathrm{C}$, $1080^{\circ} \mathrm{C}$ y $1100^{\circ} \mathrm{C}$, a cada lote de probetas se les tomo lecturas de peso y longitud en la línea de referencia, una vez alcanzaron la temperatura ambiente. "Finalmente se realizó la prueba de absorción de agua por inmersión, utilizando la metodología establecida en la norma técnica colombiana NTC 4017, la cual establece un tiempo de inmersión de 24 horas".

\section{Resultados y discusión}

En el proceso de fabricación de productos cerámicos de construcción existen una serie de variables que normalmente son controladas para asegurar la calidad de las piezas elaboradas, 
los aspectos dimensionales son una de las variables de mayor importancia, sobre todo en las piezas utilizadas como revestimientos de paredes que requieren de una tolerancia dimensional mínima, de igual manera la absorción de agua es un parámetro de gran uso que se relaciona con la porosidad de la pieza y por ende de la resistencia mecánica del material que se ha cocido.

En la industria cerámica existen diferentes materias primas que contribuyen a tener un mayor control de las variables anteriormente mencionadas, uno de estos materiales es el Carbonato de Calcio ya sea puro o el que esta contenido en las rocas calizas, Sin embargo se debe conocer plenamente las proporciones mas idóneas de material agregar, de lo contrario los resultados pueden ser problemáticos, para el caso del carbonato de calcio, la aparición de eflorescencias en la superficie de la pieza, desconchados por caliche, grietas, elevada, porosidad y aumentos energéticos durante el proceso de cocción son algunos de los efectos encontrados en fuente bibliográficas de origen español donde se establece rangos de trabajo entre 5 y $10 \%$ de carbonatos de calcio para piezas no esmaltadas [10-11], aunque suelen encontrarse rangos superiores para su uso en artículos esmaltados y generalmente utilizando la técnica de prensado para su conformado.

Las variables a las cuales se les realizó seguimiento durante la presente investigación son: contracción de secado, pérdidas de calcinación, contracción de cocción y absorción de agua, con base en la normatividad existente [12-14]. Los resultados del proceso se pueden apreciar en las figuras 2 a 5 .

Figura 2. Contracciones de secado de las probetas elaboradas

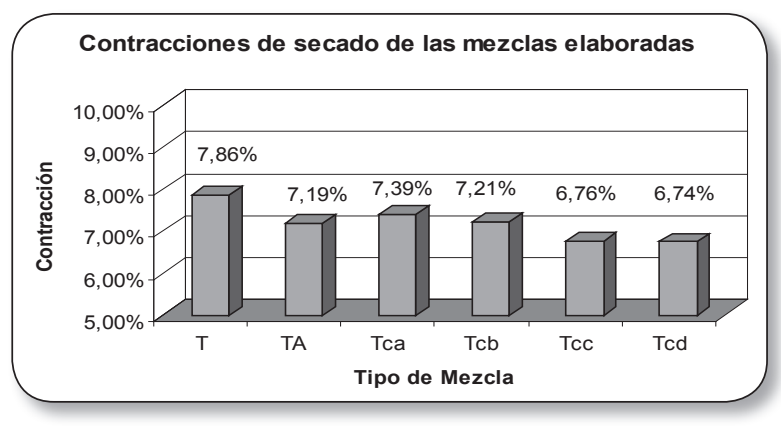

Los resultados obtenidos dejan ver una disminución de las contracciones de secado al ir aumentado el contenido de caliza adicionado, la muestra a la cual se le agregó material arenoso permite reducir las contracciones de secado, sin embargo se obtienen mejores resultados al adicionar mas del 5\% de caliza a la muestra, no se evidencia una diferencia marcada en esta variable para las diferentes mezclas que permita concluir cual resulta mas útil en esta etapa del proceso cerámico.

Figura 3. Contracción de cocción de las probetas estudiadas

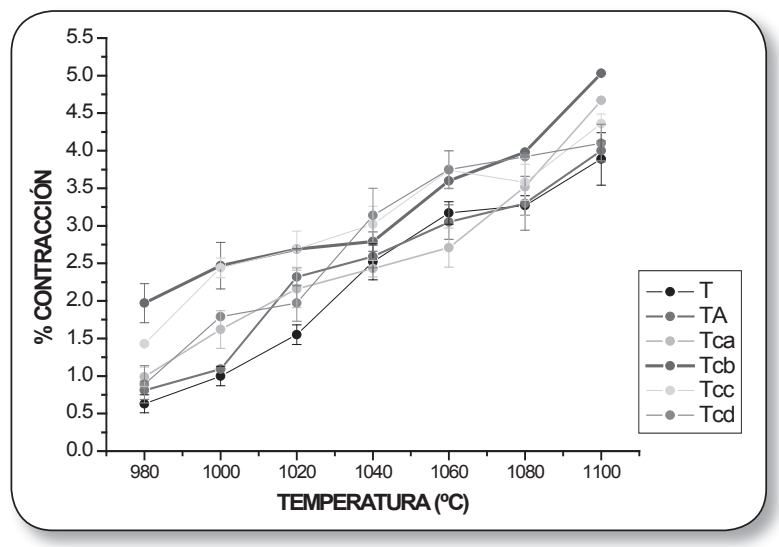

La figura 3 deja ver un aspecto muy importante referente al papel reductor de las deformaciones en la pieza por parte del carbonato de calcio en las pastas cerámicas moldeadas por extrusión, se puede apreciar que casi todas las muestras presentaron contracciones de cocción más elevadas que la muestra de arcilla natural y contrario a lo presentado en los referentes bibliográficos [2]. La explicación a este fenómeno se puede encontrar en los componentes adicionales al carbonato de Calcio que se encuentran en la caliza analizada, y que pueden actuar como fundentes como por ejemplo el oxido de Hierro y el oxido de Magnesio, como soporte a este razonamiento se encuentran los resultados de absorción de agua obtenidos. Respecto a la muestra con adición de material con alto contenido de sílice no presento diferencias significativas en comparación con la muestra de solo arcilla. 
Figura 4. Perdidas de calcinación de las probetas sometidas a cocción

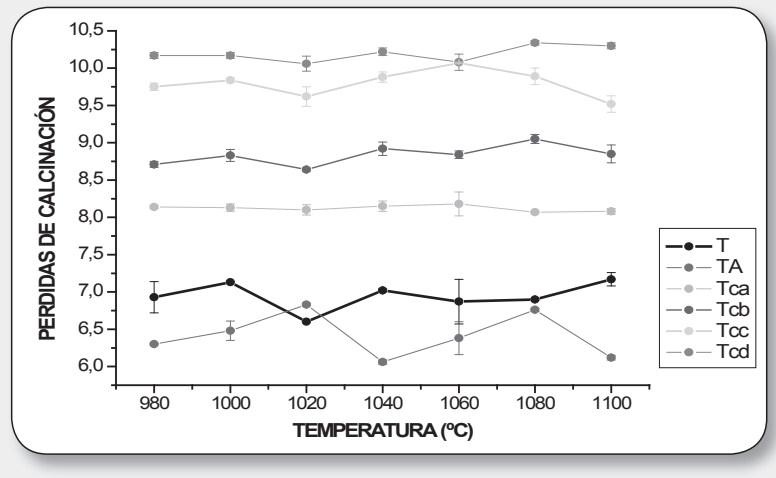

La figura 4 deja ver claramente el efecto de la descomposición de los carbonatos existentes en las probetas, el resultado de la formación de dióxido de carbono se ve reflejado en las pérdidas de calcinación, como se puede ver a mayor adición de calizas mayor son las perdidas. La muestra con adición de material con alto contenido de sílice no presenta variaciones significativas con respecto a la muestra de arcilla.

Figura 5. Absorción de agua de las probetas sometidas al proceso de cocción

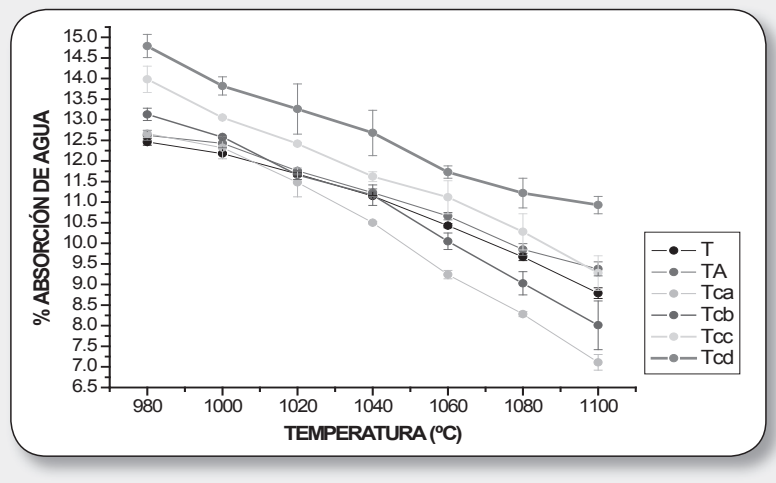

En la figura 5 se observa que la absorción de agua para las probetas con adición de caliza por debajo del $5 \%$ presentaron una absorción de agua mas baja en comparación con la muestra de arcilla pura, es decir estas probetas alcanzaron una menor porosidad, debido a una mayor vitrificación de sus componentes. La mezcla con adición de sílice no presentó diferencias significativas con respecto a la misma muestra con que se comparó las probetas de caliza (Muestra de arcilla Tapias).

\section{Conclusiones}

Los resultados de la investigación dejaron ver que la adición de calizas (La don Juana) en porcentajes inferiores al $9 \%$ y bajo el tamaño de grano agregado no contribuye significativamente al aumento de la estabilidad dimensional de las probetas elaboradas, por ello se deben realizar nuevas pruebas con otras rocas calizas de la zona para determinar si su uso realmente puede ser útil en la industria cerámica regional y si el rango de adición se puede incrementar sin causar efectos negativos en las piezas elaboradas.

En la investigación se logró determinar que la adición de calizas contribuye a reducir las contracciones de secado, y en cantidades inferiores al 5\% mejoran el proceso de fusión de las piezas en el horno por encima de los $1020^{\circ} \mathrm{C}$, esto puede llegar a ser una ventaja económica, desde el punto de vista de ahorro de combustible. Las mayores pérdidas de calcinación debido a la adición de calizas disminuyen el peso final de la pieza cocida lo cual puede resultar ventajoso, en términos de ahorros en fletes, sin embargo se deben evaluar los aspectos relacionados con la aparición de eflorescencias, tema que esta fuera del alcance de esta investigación.

\section{Agradecimiento}

Los autores expresan su agradecimiento al Centro de Investigación de Materiales Cerámicos CIMAC de la Universidad Francisco de Paula Santander por el apoyo brindado para la realización de los ensayos.

\section{Referencias Bibliográficas}


y perspectivas en la mixteca Oxaqueña. Temas de Ciencia y Tecnología, Vol 5. № 14, Pág. 3-14. En línea disponible en $<$ http://www.utm.mx/ temas/temasdocs/ensayo1t14R.pdf $>$. Tomado el 14 de Abril de 2011.

[2]. FERNÁNDEZ ABAJO, Marcelino. Manual sobre fabricación de baldosas, tejas y ladrillos [DISCO MAGNETICO]. . Laboratorio Técnico Cerámico, España. Año 2000.

[3]. JESKAR LTDA. Inventario Geológico Minero, Ambiental, Tecnológico y Empresarial de los Minerales no Energéticos del Norte de Santander. San José de Cúcuta Junio de 2000.311 P.

[4]. CARRILlO, Cárdenas Mónica y ARÉVALO, Monroy Iranny "Actualización de la información y puesta en marcha de una aplicación sistematizada para el sector cerámico del área metropolitana de san José de Cúcuta". Universidad Francisco de Paula Santander. Facultad de Ingeniería, Plan de estudios de Ingeniería de Producción Industrial. San José de Cúcuta, año 2008.

[5]. QUBIT CLUSTER TECHNOLOGY BASED CONSORTIUM. Estrategia para la conformación del Cluster y plan estratégico para la cadena de cerámica en Cúcuta. Segundo informe. Año 2007.

[6]. SÁNCHEZ MOLINA, Jorge. Diagnóstico general del sector cerámico del área metropolitana de Cúcuta. Grupo de Investigación en Tecnología Cerámica GITEC. San José de Cúcuta. Universidad Francisco de Paula Santander. Facultad de ciencias Básicas. Año 2009.

[7]. FERRO CORPORATION. Análisis químico de diferentes arcillas del área metropolitana de Cúcuta. San José de Cúcuta, Año 2001.

[8]. GELVES DIAZ, John Freddy. Desarrollo de Nuevos Productos Referente a Colores y Texturas en las Baldosas Cerámicas no Esmaltadas de la Ladrillera Sigma Ltda. Grupo de Investigación en Tecnología Cerámica
GITEC. Universidad Francisco de Paula Santander. 2010.

[9]. RIVERA CARVAJAL, Pedro Saul; ASELA MOROS, José Enrique, SUÁREZ ACEVEDO, Yohana y ZÚNIGA ROJAS, Carlos Alberto. Evaluación de calizas para la obtención de una cal para el suavizamiento de aguas subterráneas a nivel de laboratorio, utilizadas para consumo humano en el municipio de Villa del Rosario, departamento Norte de Santander. RESPUESTAS - Año 12 No. 1 - Julio 2007 ISSN 0122820X.

[10]. CENTRO DE TECNOLOGÍA DE LA ARCILLA COCIDA Y LA ASOCIACIÓN PARA LA INVESTIGACIÓN Y EL DESARROLLO INDUSTRIAL DE LOS RECURSOS NATURALES. Manual de patologias de las piezas cerámicas para la construcción. Toledo, España, 1999.

[11]. ASOCIACIÓN PARA LA INVESTIGACIÓN Y Y DESARROLLO INDUSTRIAL DE LOS RECURSOS NATURALES AITEMIN. Aditivos utilizados en la industria cerámica estructural. Memorias primer curso internacional de cerámica, San José de Cúcuta, Universidad Francisco de Paula Santander, 2005.

[12]. INSTITUTO COLOMBIANO DE NORMAS TÉCNICAS Y CERTIFICACIÓN, NTC 4017 Métodos para muestreo y ensayos de unidades de mampostería y otros productos de arcilla. Bogota D.C. ICONTEC, 2005.

[13]. INSTITUTO COLOMBIANO DE NORMAS TÉCNICAS Y CERTIFICACIÓN, NTC 1495. Suelos. Ensayo para determinar el contenido de humedad de suelos y rocas, con base en la masa. Bogota D.C. ICONTEC, 2005.

[14]. AMERICAN SOCIETY FOR TESTING AND MATERIALS. ASTM C 326-03. Determinación de la contracción Lineal en seco y por quema. 